\title{
IMPLICAÇÕES NO CUIDADO DE ENFERMAGEM À CRIANÇA COM CÂNCER HOSPITALIZADA
}

\author{
IMPLICATIONS ON THE NURSING CARE OF HOSPITALIZED CHILDREN WITH CANCER
}

\section{Kálya Yasmine Nunes de Lima ${ }^{{ }^{*}}$, Viviane Euzébia Pereira Santos ${ }^{b^{*}}$, Francis Solange Vieira Tourinho ${ }^{c^{*}}$}

\author{
alima.yasmine@yahoo.com.br, bvivianeepsantos@gmail.com, cfrancistourinho@gmail.com \\ *Universidade Federal do Rio Grande do Norte
}

Data de entrega do artigo: 21/10/2013

Data de aceite do artigo: 19/03/2014

\section{RESUMO}

Introduçáo: Cuidar de crianças com câncer em unidades oncológicas demanda dos profissionais conhecimento técnico-científico, disponibilidade, flexibilidade, reconhecimento de limites, posturas éticas e capacidade de interação com a criança e sua família. Objetivo: Analisar os aspectos contextuais do cuidado de enfermagem à criança hospitalizada para tratamento do câncer. Métodos: Revisão integrativa realizada nas bases de dados Scopus, National Library of Medicine National Institutes of Health (PubMed), Cumulative Index to Nursing and Allied Health Literature (CINAHL), Scientific Electronic Library Online (SciELO) e Literatura Latino-Americana e do Caribe em Ciências da Saúde (LILACS). A amostra final resultou em 22 artigos. Para apreciação dos dados, empregou-se a análise de contexto proposta por Hinds, Chaves e Cypress. Resultados: No contexto imediato, abordou-se como ocorre o cuidado de enfermagem por meio da categoria "Interação profissional-paciente-família no processo de cuidar". Para o contexto específico, elencou-se a categoria "Dificuldades que comprometem o cuidado de enfermagem". O contexto geral foi destacado pela categoria "Estigmas da doença" e, por fim, o metacontexto trouxe uma perspectiva global sobre atenção oncológica por meio da categoria "O cuidado orientado pelas políticas de saúde". Conclusáo: $\mathrm{O}$ estudo trouxe uma reflexáo sobre os fatores que influenciam no cuidado de enfermagem à criança com câncer hospitalizada a partir de uma análise dos contextos que permeiam a sua prática. O cuidado à criança segue ainda uma perspectiva mecanicista, fragmentada e biologicista, a qual é influenciada pela falta de preparo do profissional para lidar com a situação, a própria normatização da instituição e as crenças negativas acerca da doença oncológica.

Palavras-chave: Enfermagem; enfermagem oncológica; criança hospitalizada.

\section{ABSTRACT}

Introduction: Taking care of children with cancer in cancer units demands professionals with technical and scientific knowledge, availability, flexibility, recognition of limits, ethical stances and ability to interact with the child and family. Objective: To analyze the contextual aspects of nursing care for hospitalized children with cancer. Method: Integrative review held in the databases Scopus, National Library of Medicine National Institutes of Health (PubMed), Cumulative Index to Nursing and Allied Health Literature (CINAHL), Scientific Electronic Library Online (SciELO) and Literature Latin American and Caribbean Health Sciences (LILACS). The final sample resulted in 22 articles. To evaluate the data, it was used a contextual analysis proposed by Hinds, Chaves and Cypress. Results: In the immediate context it was addressed how nursing care occurs by the category "Professional-patient interaction in family-care process". For the specific context it was listed the category "Difficulties undertake nursing care." The 
general context was highlighted by the category "Stigmata disease" and, finally, the metacontext brought a global perspective on cancer care with the category "Care-oriented health policies". Conclusion: The study carried a reflection on the factors that influence the nursing care of hospitalized children with cancer from the analysis of contexts that permeate their practice. The child care also follows a mechanistic, fragmented and biologicist perspective, which is influenced by the lack of professional preparation to deal with the situation, the standards of the institution and negative beliefs about cancer disease.

Keywords: Nursing; oncologic nursing; hospitalized child.

\section{Introdução}

O câncer na infância já foi considerado uma doença aguda e de evolução invariavelmente fatal, constituindo-se numa das principais causas de morte no Brasil ${ }^{1}$. Hoje, no entanto, já é considerado o tipo de câncer com mais perspectiva de cura, sendo avaliado, na maioria dos casos, como de bom prognóstico.

O diagnóstico da doença e o processo de hospitalização provocam estresse na família e na criança, as quais se deparam com conceitos já formados acerca da doença, provocando impactos nesses indivíduos. Geralmente, ocorrem os momentos de protesto, de desespero e de negação diante do diagnóstico. Muitos pais tendem a se culpar pela situação e a criança sofre também com todas as consequências da doença ${ }^{(2)}$.

Nesse sentido, o cuidar de crianças com câncer em unidades oncológicas representa um desafio às competências do profissional, demandando destes, além do conhecimento técnico-científico, disponibilidade, flexibilidade, reconhecimento de limites e posturas éticas e capacidade de interação, competências estas imprescindíveis para o sucesso no tratamento e no relacionamento entre a equipe de saúde, em especial de enfermagem, as crianças e seus familiares ${ }^{3}$.

O enfermeiro deve compreender que crianças e adultos diferem quanto ao modo de vivenciar uma doença como o câncer, e, assim, também possuem demandas de cuidado diferentes. Cabe, então, ao profissional sensibilidade e envolvimento com a criança para compreender o que é indispensável na visão dela para a concretizaçáo do cuidado de forma plena, englobando tanto os aspectos físicos quanto os psicossociais.

Deste modo, a qualidade do cuidado prestado pela equipe de enfermagem se beneficia quando atenta para o que permeia o universo infantil, viabilizando a resolução de problemas, estreitando vínculos e construindo corresponsabilidades, contribuindo para atenção e satisfação das necessidades singulares de cada momento vivenciado por essas crianças e suas famílias, visando solidariedade, ética e equipes de trabalho que respeitam e valorizam as diferenças com responsabilidades, compromissos e metas ${ }^{3}$.

No entanto, a literatura aponta que existem muitas dificuldades para se estabelecer o cuidado de enfermagem adequado a criança hospitalizada para tratamento do câncer, que vão desde o despreparo para lidar com a situação até a supervalorização dos procedimentos técnicos em detrimento do cuidar mais relacional e próximo da criança e sua família ${ }^{4}$.

Ainda que a enfermagem seja uma profissão que visualiza o cuidar por um processo de interação, de proximidade e de preocupação com o outro, buscando o alívio do sofrimento, qualidade de vida e auxílio ao indivíduo a lidar com os momentos de crise ${ }^{5}$, o que se observa é que o cuidado ainda possui características do modelo biomédico, voltado para açôes curativas e fragmentadas, sem considerar a complexidade do que é adoecer de câncer enquanto criança ${ }^{6}$.

Levando em consideração que o cuidado de enfermagem pode sofrer influências de diversas origens, sejam as relacionadas às características pessoais até aquelas vinculadas ao contexto social e político da sociedade, elencou-se o seguinte questionamento: em quais contextos ocorre o cuidado de enfermagem à criança hospitalizada com câncer? Assim, o presente estudo tem por objetivo analisar os aspectos contextuais do cuidado de enfermagem à criança hospitalizada para tratamento do câncer.

Dessa forma, espera-se contribuir para uma maior compreensão sobre o cuidado de enfermagem à criança hospitalizada para tratamento do câncer e sua relaçáo com os diferentes contextos existentes no processo de trabalho do enfermeiro, visando suscitar a reflexão e o desenvolvimento de novas práticas que ofereçam um cuidado de enfermagem mais humanizado e de qualidade à criança em tratamento oncológico.

\section{Métodos}

Este estudo foi realizado por meio da revisão integrativa, a qual se constitui num método que proporciona reunir e sintetizar o conhecimento produzido sobre um delimitado tema ou questão de pesquisa ${ }^{7}$.

A busca foi realizada no mês de julho de 2013, nas bases de dados: Scopus, National Library of Medicine National Institutes of Health (Pubmed), Cumulative Index to Nursing and Allied Health Literature (CINAHL), Scientific Electronic Library Online 
(SciELO) e Literatura Latino-Americana e do Caribe em Ciências da Saúde (LILACS), utilizando-se os Descritores em Ciências da Saúde (DeCS), enfermagem oncológica, cuidados de enfermagem e criança e a versão dessas palavras na língua inglesa nas bases de dados internacionais.

Os critérios de inclusão dos estudos foram: artigos publicados em português, inglês ou espanhol, com textos completos disponíveis gratuitamente, que abordassem os aspectos contextuais do cuidado de enfermagem à criança com câncer hospitalizada.

A amostra final resultou em 22 artigos, conforme especificado no quadro1.

Quadro 1: Distribuição do quantitativo de estudos de acordo com a base de dados. Natal, RN, 2013.

\begin{tabular}{cc}
\hline Base de dados & Quantitativo de estudos \\
\hline SciELO & 7 \\
LILACS & 6 \\
CINAHL & 4 \\
Pubmed & 4 \\
Scopus & 1 \\
Total & 22 \\
\hline
\end{tabular}

Fonte: dados da pesquisa.

Para apreciação dos dados, empregou-se a análise de contexto proposta por Hinds, Chaves e Cypress ${ }^{8}$ para compreensão de um fenômeno.

Nesta proposta, o contexto é considerado o cenário no qual ocorre o fenômeno, ponderando todos os fatores que influenciam e influenciaram em sua ocorrência. Ele é constituído por quatro camadas interativas, que se distinguem entre si e que vão desde o significado individualizado ao universal, a saber: o contexto imediato, no qual se definem os aspectos da situação, tais como espaço onde o ato ocorre e seus limites; o específico, o qual tem por principal característica o conhecimento individualizado e único que engloba o passado imediato e os aspectos relevantes da presente situação; o geral, que se baseia pelo quadro de referências de vida desenvolvido a partir de interpretaçóes obtidas de interaçóes passadas e atuais; e o metacontexto que se configura é uma fonte de conhecimento socialmente construído que opera e que resulta em uma perspectiva social compartilhada ${ }^{8}$.

\section{Resultados e discussão}

Por meio da reflexão apoiada na revisão integrativa, na discussão do contexto imediato, abordou-se o fenômeno do cuidado a partir das especificidades da assistência de enfermagem à criança com câncer hospitalizada e como ele vem ocorrendo. Assim, neste estudo, essa camada de contexto será denominada "Interação profissional-paciente-família no processo de cuidar".

No contexto específico, buscou-se descrever os aspectos individuais, ambientais e do processo de trabalho do enfermeiro que dificultam o cuidado de enfermagem adequado à criança hospitalizada, por meio da categoria "Dificuldades que comprometem o cuidado de enfermagem".

Para o contexto geral, destacaram-se os fatores que permeiam a prática dos profissionais de enfermagem, no que se referem a crenças, valores, hábitos, sentimentos e experiências vivenciadas no exercício de sua profissão que influenciam no cuidado oferecido. Deste modo, esse contexto terá a denominação "Estigmas da doença".

E no metacontexto, abordaram-se as políticas de saúde como guia para a formação e atuação de profissionais na assistência oncológica, por meio da categoria " $\mathrm{O}$ cuidado orientado pelas políticas de saúde”.

\section{Interação profissional-paciente-família no processo de cuidar}

Como constituinte do primeiro nível contextual em análise, o cuidado de enfermagem, indicado na literatura, surge a partir do cuidar, o qual é profissionalizado, demandando conhecimento técnico científico, que constitui o corpo de conhecimento próprio da enfermagem, sendo essa práxis envolvida em respeito, compreensão e responsabilidade com a saúde de si e do outro. Deste modo, o cuidado de enfermagem requer conhecimento, habilidades e atitudes?

$O$ cuidado de enfermagem destinado à criança com câncer hospitalizada ainda está voltado para realização de procedimentos técnicos, com enfoque mecanicista, atendendo basicamente as necessidades do corpo biológico da criança, não a considerando como um ser em crescimento e desenvolvimento com determinaçôes familiares, culturais, ambientais e econômicas ${ }^{4,10-13}$.

$\mathrm{O}$ enfermeiro busca não se envolver com o paciente, e com isso mantém uma distância considerada "segura" por ele mesmo para náo sofrer com as situaçóes vivenciadas pela criança ${ }^{4,14}$, o que por sua vez prejudica também o processo comunicativo entre enfermeiro e paciente.

Nesse sentido, há questionamentos e dificuldades no que se refere à comunicação, ao envolvimento e ao atendimento das necessidades emocionais da criança e de seus familiares. A literatura aponta que o enfermeiro, por vezes, se perde na tentativa de estabelecer uma distância considerada adequada da criança, prevalecendo, então, o atendimento apenas das necessidades físicas $^{15,16}$.

Por outro lado, tem se observado uma nova tendência no cuidado de enfermagem em oncologia pediátrica, 
que está voltada para a participação da família no processo de cuidar da criança ${ }^{2}$.

Durante a hospitalização, a família é considerada o ponto de apoio do paciente e diante de todas as mudanças em sua rotina e situaçóes estressantes, ela precisa também ser foco do cuidado de enfermagem e ser incorporada no cuidado à criança.

Contudo, embora os enfermeiros já reconheçam a importância da família e a necessidade de se destinar cuidados para ela e de envolvê-la no cuidado dedicado à criança, eles ainda sentem dificuldades em lidar com a presença dos parentes, chegando ao ponto de afirmarem que evitam ao máximo o contato com os familiares das crianças internadas ${ }^{4}$, pelo anseio de se estabelecer vínculos afetivos e criar sentimentos em relação a eles e sua criança.

$\mathrm{O}$ que se reflete em cuidadores que desconhecem o quadro real da criança, e que não participam de forma efetiva no planejamento e aplicação de medidas que promovam o cuidado, assim como dificuldades de comunicação entre familiares, crianças e enfermeiros, o que favorece o cuidado centrado na doença e sem levar em consideração o contexto em que a criança está inserida, ou seja, sua família.

Frente a isso, o cuidado à criança hospitalizada é permeado por medos, angústias e distanciamento entre profissionais, crianças e familiares, sendo enfatizados os procedimentos técnicos direcionados às necessidades biológicas da criança.

\section{Dificuldades que comprometem o cuidado de enfermagem}

A enfermagem, ao longo dos anos, vem assumindo diversos papéis nos setores hospitalares, que muitas vezes não correspondem à essência de sua profissão. Além disso, as atividades burocráticas do serviço consomem maior parte do tempo dos enfermeiros, que, devido a isso, não conseguem interagir com a criança e sua família para o planejamento do cuidado.

Vale destacar que o excesso de atividades e o constante desenvolvimento tecnológico, refletido no grande aparato maquinário existente no hospital, demonstram que os profissionais, por vezes, acabam adotando atitudes impessoais, robóticas e se ajustam às pressôes administrativas que priorizam a produtividade e o desempenho ${ }^{17}$, deixando um pouco de lado a sensibilidade do cuidado e o envolvimento com o paciente.

Diante disso, a literatura destaca certa invisibilidade da real função do enfermeiro no hospital. Suas ações são mais identificadas, rotineiramente, por uma forte ligação a execução de atividades burocráticas. A atuação desse profissional está fortemente associada à ideia de fazer de tudo um pouco, distanciando-se, com isso, da sua função principal, que é a assistência ao paciente a partir das suas necessidades específicas ${ }^{12}$.

A falta de preparo durante a formação profissional e de capacitaçóes e treinamentos por parte da instituição empregadora sobre o cuidado a criança oncológica, também são vistos como fatores que dificultam o cuidado. Estes profissionais sentem-se inseguros e despreparados para lidar com as situaçóes inerentes à criança com câncer, com isso, apontam que preferem realizar as atividades burocráticas do serviço do que se envolver com ela e sua famíliaa, ${ }^{40,14,15,18-20}$.

Embora existam algumas iniciativas institucionais de se realizar capacitaçôes e atualizaçôes, elas não são feitas de forma integrada com os enfermeiros que lidam com cuidado à criança com câncer, nesse sentido, tem-se um caráter de educação continuada, em que os profissionais não são participativos em propor mudanças para os problemas oriundos da prática, com isso, muitos entraves relacionados ao processo de trabalho do enfermeiro ainda persistem nos setores de oncologia pediátrica ${ }^{3}$.

O cuidado é influenciado também pelo salário oferecido, que é a fonte de sobrevivência do trabalhador; e pela necessidade de se mostrar sempre como um profissional que não sofre diante da situação de seu paciente, e que, portanto, não deixa seu lado emocional transparecer em suas relaçóes profissionais ${ }^{4}$. Nessa perspectiva, cria-se a impossibilidade de incluir, na rotina, sentimentos, desejos e necessidades envolvidos nas relações. Com isso, a assistência sofre influência do silenciamento que a organização da instituição impóe à criatividade dos profissionais, na rigidez das hierarquias estabelecidas, na negação dos conflitos inerentes à vida ${ }^{10}$.

Deste modo, observa-se uma classe profissional que sofre e que, ao mesmo tempo, se adéqua, com as questôes organizacionais e modelos assistenciais existentes nos estabelecimentos de saúde e que ainda não possui seu reconhecimento profissional por meio de um salário digno, o que gera desgaste emocional e físico, causados pelos múltiplos vínculos empregatícios, que os enfermeiros assumem para compensar a baixa remuneração.

Por outro lado, existe a dificuldade de lidar com os sentimentos e cargas emocionais advindos da situaçáo, que, muitas vezes, são negligenciados pelas instituiçôes. Cuidar de crianças em setores de oncologia gera sofrimentos no profissional e, frente a isso, os estabelecimentos de saúde devem oferecer apoio e medidas que o auxiliem a superar os sentimentos negativos e a lidar com as situaçóes que surgem nesse ambiente ${ }^{6}$, gerando qualidade de vida também para o enfermeiro, para que ele possa estar apto a oferecer um cuidado humanizado e integral. 


\section{Estigmas da doença}

Assistir o paciente com câncer traz significados diversos, mudanças de valores, crenças e atitudes que demandam intervençóes apropriadas e individualizadas para minimizar os efeitos negativos na doença e terapêutica, o que leva o profissional, a confrontar-se com sua própria vulnerabilidade e finitude $\mathrm{e}^{3,20}$.

Mesmo com o avanço no tratamento do câncer e de sua ocorrência em crianças ser considerada de bom prognóstico, na maioria dos casos, ele ainda é uma doença que carrega consigo o estigma da morte e sofrimento que impera no imaginário social.

A relação estabelecida entre câncer e morte, decorrente das concepçóes errôneas acerca da doença, contribui para um comportamento de fuga das pessoas, inclusive dos profissionais ${ }^{4,20,21}$.

Não obstante, o câncer na criança, mais intensamente do que no adulto, determina expressóes de pena e pesar, em razão do medo e mito da doença oncológica. Há sofrimento advindo do envolvimento com a criança e sua família e da impotência frente à possibilidade de evolução negativa da doença, o que gera sensaçóes de impotência, insuficiência, culpa, desprezo, tristeza, podendo resultar em atendimento frio e impessoal, fuga às perguntas do paciente e aos seus pedidos de socorro ${ }^{10,15}$.

Por mais que a equipe de enfermagem se faça mais presente do que outros profissionais, ela costuma apresentar dificuldades em lidar com as emoçóes e ideias expressas pelos pacientes em consequência da preparação insuficiente e do envolvimento emocional mais significativo.

Importante ressaltar que os valores e posicionamentos do enfermeiro frente à criança com câncer são provenientes de uma mescla de fatores que abrange a formação às vivências na prática, incluindo a subjetividade dos profissionais e os resquícios da história da profissão de enfermagem ${ }^{12}$. $\mathrm{O}$ que cada enfermeiro traz para qualquer encontro em saúde envolve a base única de conhecimentos adquiridos ao longo da vida, das experiências, das interações anteriores, reflexão e empatia $^{20,22}$.

Nesse sentido, levando em consideração a história e a atual situação do câncer infantil, nota-se que devido à ausência do diagnóstico precoce e o difícil acesso aos serviços de saúde especializados, muitas crianças chegam para o tratamento no hospital em estágios muito avançados da doença, nos quais a recuperação torna-se difícil ${ }^{23}$. Com isso, o profissional está habituado a receber crianças com câncer em fases críticas e que, consequentemente, evoluem para óbito, o que pode justificar, em parte, porque a doença ainda está muito ligada à visão de morte e sofrimento para os enfermeiros.
Frente a isso, existe ainda a falta de capacitação para lidar com os aspectos subjetivos que envolvem o cuidado de enfermagem, a finitude da vida e, principalmente, de atualizaçóes que abordem todas as possibilidades de tratamento e de cuidado de enfermagem para a criança com câncer.

Independente da gravidade, do estágio e das experiências anteriores do enfermeiro, é relevante lembrar que, evoluindo para óbito ou para cura, a criança com câncer necessita de cuidados especializados, que podem se prolongar por meses ou até anos, e que os profissionais precisam encontrar formas de amenizar o sofrimento vivenciado por ela e sua família ${ }^{17}$.

\section{O cuidado orientado pelas políticas de saúde}

No Brasil e no mundo, o câncer pediátrico e juvenil é considerado um problema de saúde pública ${ }^{4,11,24}$. Frente a isso, a proposta brasileira de política pública para o câncer na infância baseia-se, principalmente, no diagnóstico precoce, objetivando aumentar as taxas de sobrevida das crianças.

A terapêutica do câncer envolve as fases de diagnóstico, modalidades de tratamento e controle, e tem dois objetivos: aumentar as taxas de sobrevida, minimizando os efeitos tardios do tratamento, e reintegrar a criança na sociedade com qualidade de vida ${ }^{14}$.

Para tanto, a Política Nacional de Atenção Oncológica (PNAO), instituída em 2005, estruturase em redes estaduais e regionais, na lógica da linha de cuidados progressivos. Diante disso, ressalta-se a importância dos sistemas de referência e contra referência, visando à integralidade da assistência por meio do acesso aos diferentes níveis de atenção, de acordo com a necessidade da criança e a continuidade do cuidado ${ }^{25}$.

Nessa perspectiva, a alta complexidade tem o papel de garantir o acesso dos doentes com diagnóstico clínico ou com diagnóstico definitivo de câncer a esse nível de atenção, determinar a extensão da neoplasia (estadiamento), tratar, cuidar e assegurar qualidade de acordo com rotinas e condutas estabelecidas, assistência essa que se dará por meio de Unidades de Assistência de Alta Complexidade em Oncologia e Centros de Assistência de Alta Complexidade em Oncologia $(\mathrm{CACON})^{25}$.

Entre os eixos norteadores da política, estão a qualificação da assistência e a promoção da educação permanente dos profissionais de saúde envolvidos com a sua implantação e implementação, em consonância com os princípios da integralidade e da humanização ${ }^{25}$.

A educação permanente nos setores de oncologia pediátrica visa à emancipação dos profissionais em relação a identificação dos problemas e suas propostas de resolução para melhoria do cuidado de enfermagem ${ }^{26}$, 
assim, o enfermeiro é visto como um transformador de sua realidade, promovendo a interlocução entre as diversas esferas gestoras e assistenciais.

Nesse sentido, a PNAO enfatiza a especialização, qualificaçáo e educação permanente dos profissionais para o controle do câncer. Constitui-se, entâo, na proposta de criação de uma Rede de Educação Permanente em Atenção Oncológica, com capacidade de reforçar e ampliar a vinculação entre trabalho, gestáo e educaçáo, no contexto do Sistema Único de Saúde e na perspectiva dos princípios norteadores desse sistema ${ }^{25}$.

Importante destacar que, embora exista uma política que permita o credenciamento das instituiçóes de saúde que se responsabilizarão pela assistência a criança com câncer nos diferentes níveis de atenção, a realidade mostra que, em relação à internação em nível hospitalar, muitas instituiçôes que fazem o tratamento não são credenciadas e, portanto, não recebem o repasse financeiro para a implementação das diferentes modalidades terapêuticas e capacitação de seus profissionais ${ }^{27}$. Essa situação é o reflexo de uma política que ainda não está sendo colocada em prática como se determina.

Contudo, mesmo diante dos entraves na prática, a atenção a criança com câncer defende a aplicação de esforços em busca da qualificação dos profissionais, principalmente, no que se refere ao diagnóstico precoce e novos tratamentos. Frente a isso, existe um grande incentivo às pesquisas e produção de conhecimento na área, objetivando a busca por conhecimento e a melhoria da assistência.

O que deixa claro que, para obter sucesso com as açôes de saúde, se faz necessário o conhecimento sobre a política que envolve o câncer infantil, assim como a identificação dos aspectos biológicos, psicossociais e de cuidado, peculiares ao contexto do câncer infantil ${ }^{23}$.

Nota-se, portanto, que o cuidado à criança com câncer vem sendo reestruturado numa perspectiva integral, com base na transdisciplinaridade e qualificação profissional. Deste modo, o conhecimento socialmente construído e compartilhado visa à superação do modelo biomédico em busca de um cuidado complexo que envolva todos os aspectos inerentes ao adoecer de câncer.

\section{Conclusão}

O estudo trouxe uma reflexão sobre os fatores que influenciam no cuidado de enfermagem à criança com câncer hospitalizada a partir de uma análise dos diferentes contextos que permeiam a sua prática.

Constatou-se que o cuidado ao público infantil não ocorre de acordo com o que é defendido pela profissão, seguindo ainda uma perspectiva mecanicista, fragmentada e biologicista, a qual é influenciada pela falta de preparo do profissional para lidar com a situação e a própria normatização da instituição que impede, muitas vezes, que este tenha uma relação mais próxima com a criança e sua família para o planejamento e execução do cuidado.

As crenças sobre a doença oncológica, advindas da própria experiência profissional e do desconhecimento sobre os novos tratamentos e perspectivas de cura, por sua vez, reforçam o afastamento do profissional e da criança, pelo receio da morte.

Diante disso, destaca-se o papel da educação permanente defendida pela PNAO como importante estratégia para superaçẫo dos entraves encontrados na prática do cuidado à criança oncológica. Mas também é necessário que a enfermagem reflita sua prática e invista esforços para o resgate do cuidado relacional, humanizado e integral.

Nesse sentido, a enfermagem deve deixar claro qual o seu papel diante do cuidado à criança com câncer nos diferentes níveis de atenção, assim como nas instituiçôes de ensino e nos serviços de saúde, proporcionando a atuação de enfermeiros comprometidos com a qualidade do cuidado oferecido e com os seus direitos frente às necessidades que surgem na assistência em oncologia pediátrica.

\section{Referências}

1. Beltrao MR, Vasconcelos MG, Pontes CM, Albuquerque MC. Childhood cancer: maternal perceptions and strategies for coping with diagnosis. J Pediatr. 2007; 83(6): 562-66.

2. Avanci BS, Carolindo FM, Góes FGB, Netto NPC. Cuidados paliativos à criança oncológica na situaçáo do viver/morrer: a ótica do cuidar em enfermagem. Esc Anna Nery R Enferm. 2009; 13(4): 708-16.

3. Amador DD, Gomes IP, Coutinho SED, Costa TNA, Collet N. Concepção dos enfermeiros acerca da capacitação no cuidado à criança com câncer. Texto Contexto Enferm. 2011; 20(1): 94-101.

4. Oliveira MCL, Firmes MPR. Sentimentos dos profissionais de enfermagem em relação ao paciente oncológico. REME. 2012; 16(1): 91-7.

5. Waldow VR. Cuidar: Expressão humanizadora da enfermagem. 2 ed. Petrópolis: Vozes; 2007.

6. Salimena AMO, Teixeira SR, Amorim TV, Paiva ACPC, Melo MCSC. Estratégias de enfrentamento usadas por enfermeiros ao cuidar de pacientes oncológicos. Rev Enferm UFSM. 2013; 3(1): 8-16.

7. Mendes KDS, Silveira RCCP, Galvão CM. Revisão integrativa: método de pesquisa para a incorporação de evidências na saúde e na enfermagem. Texto Contexto Enferm, Florianópolis. 2008; 17(4): 758-64.

8. Hinds PS, Chaves D, Cypress SM. Context as a source of meaning and understanding. Qual Heath Res. 1992; 2(1): $61-74$. 
9. Waldow VR. O cuidado na saúde: as relaçôes entre o eu, o outro e o cosmos. Petrópolis: Vozes; 2004.

10. Paro D, Paro J, Ferreira DLM. O enfermeiro e o cuidar em oncologia pediátrica. Arq Ciênc Saúde. 2005;12(3): 121-57.

11. Lima RAG, Sochi CGS, Kamada I, Rocha SMM. Assistência à criança com câncer: análise do processo de trabalho. Rev Esc Enferm USP. 1996; 30(1): 14-24.

12. Backes DS, Cruz EA. Planejamento da assistência de enfermagem ao paciente com câncer: reflexão teórica sobre as dimensões sociais. Esc Anna Nery R Enferm. 2011; 15(1): $180-5$.

13. Hawes R. Therapeutic relationships with children and families. Pediatric Nurs. 2005; 17 (6): 15-8.

14. Mutti CF, Padoin SMM, Paula CC. Espacialidade do ser-profissional-de-enfermagem no mundo do cuidado à criança que tem câncer. Esc Anna Nery R Enferm. 2012; 16(3): 493-9.

15. Françoso LPC. Reflexóes sobre o preparo do enfermeiro na área de oncologia pediátrica. Rev latinoam enferm. 1996; 4(3): 41-48.

16. Vatne TM, Slaugther L, Ruland CM. How children with câncer communication and think about symptoms. J Pediatr Oncol Nursing. 2010; 27(1): 24-32.

17. Waldow VR. Bases e princípios do conhecimento e da arte da enfermagem. Petrópolis: Vozes; 2008.

18. Romaniuk DK, Kristjanson LJ. The parent-nurse relationship from the perspective of parents of children with câncer. J Pediatr Oncol Nursing. 1995; 12(2): 80-9.

19. Barranco E, Moreira MC, Menezes MFB. O líder de enfermagem em unidades oncológicas: intervençôes da subjetividade na organização de espaços saudáveis de trabalho. Rev bras cancerol. 2010; 56(2): 213-18.
20. Silva RCV, Cruz EA. Planejamento da assistência de enfermagem ao paciente com câncer: reflexão teórica sobre as dimensôes sociais. Esc Anna Nery R Enferm. 2011; 15(1): 180-5.

21. Melo LL, Valle ERM. Equipe de enfermagem: experiência do cuidar de criança com câncer nos plantóes noturnos. Rev Esc Enferm USP. 1998; 32(4): 325-34.

22. Cantrel MA. The art of pediatric oncology nursing practice. J Pediatr Oncol Nursing.2007; 24(3): 132-8.

23. Azevedo MCCV, Lima KYN, Santos ADB, Monteiro AI. Team's performance in primary health care on early identification of children's câncer. Rev pesqui cuid fundam. 2012; 4(3): 2692-01

24. Lima RAG. Políticas de controle do câncer infantojuvenil no Brasil. Rev Latinoam Enferm. 2009;17(6): 927-8.

25. Ministério da Saúde. Portaria no 2.439 , de 8 de dezembro de 2005. Institui a Política Nacional de Atenção Oncológica: Promoção, Prevenção, Diagnóstico, Tratamento, Reabilitação e Cuidados Paliativos, a ser implantada em todas as unidades federadas, respeitadas as competências das três esferas de gestão. Diário Oficial União. 9 dez 2005. Seção 1.

26. Vincent SP. Educação permanente: componente estratégico para a implementação da política nacional de atenção oncológica. Rev Bras Cancerol. 2007; 53(1): 79-85.

27. Azevedo MCCV. Identificação de crianças e adolescentes com suspeita de câncer: uma proposta de intervençáo [dissertação]. Natal (RN): Programa de Pós-graduação em enfermagem, Universidade Federal do Rio Grande do Norte; 2010 . 